\title{
Operationen am kindlichen Ellbogen
}

\author{
Peter Ferlic, Silvia Zötsch, Annelie Weinberg
}

\section{Zusammenfassung}

Frakturen im Bereich des Ellbogens machen in etwa 7-17\% aller Frakturen im Kindesalter aus. Das Ellbogengelenk ist sowohl in seiner Anatomie, mit 3 beteiligten Knochen, als auch in seiner Funktion ein komplexes Gelenk. In Abhängigkeit vom Unfallmechanismus ist eine Vielzahl unterschiedlicher Verletzungsmuster beschrieben, welche von einfachen Knochenbrüchen bis $\mathrm{zu}$ schweren Luxationsfrakturen reichen können. Eine konventionell radiologische Bildgebung ist meist wegweisend. Aufgrund der engen Beziehung zu den Gefäßen und Nerven müssen eine periphere Durchblutungsstörung oder ein neurologisches Defizit immer ausgeschlossen werden. Aufgrund der nur geringen Wachstumspotenz der Wachstumsfuge am distalen Humerus sind spontane Achskorrekturen nur in einem geringen Ausmaß zu erwarten. Entsprechend muss eine anatomische
Reposition Ziel jeder Behandlung von Humerusfrakturen sein, um einer langfristigen Funktionsstörung der oberen Extremität vorzubeugen. Kann diese mit konservativen Maßnahmen nicht erzielt werden, ist eine operative Therapie indiziert. Am proximalen Unterarm ist die Korrekturpotenz wesentlich besser und kann in die Therapie integriert werden. In Abhängigkeit der Lokalisation der Verletzung können unterschiedliche Osteosyntheseverfahren zum Einsatz kommen. Deren Indikationen und die operativen Techniken sollen in diesem Artikel aufgearbeitet werden.

\section{Operations on the Paediatric Elbow}

Fractures of the elbow add up to about $7-17 \%$ of all fractures in childhood. The elbow joint has a complex anatomy, with 3 bones linked together, that allows a wide range of motion and is fundamental for full function of the upper extremity. Depending on the mechanism of trauma, a wide variety of injuries have been described, ranging from simple fractures to severe luxation injuries. Conventional $\mathrm{x}$-ray imaging in two plains allows an adequate diagnosis in most cases. However, impairment of peripheral blood perfusion and neurological deficits have to be excluded, due to the close proximity of nerves and blood vessels. The growth plate of the distal humerus accounts only slightly for the growth of length, therefore spontaneous correction of malalignment can only be expected to a small extent. Consequently, anatomic reduction must be achieved with the treatment to preserve full function of the arm. If this is not guaranteed with conservative interventions, surgical treatment can be indicated. Depending on the location of the injury different methods of osteosynthesis can be applied. The surgical techniques and their indication are put in focus in this paper.

\section{Einleitung}

Im Kindesalter machen die Ellbogenfrakturen $7-17 \%$ aller Frakturen aus und zählen somit zu den häufigsten Frakturen im Wachstumsalter [1]. Der hohen Inzidenz entsprechend wird auch regelmäßig eine operative Versorgung notwendig. Die Kenntnis der anatomischen Grundlagen und Behandlungsstrategien ist daher für alle beteiligten Berufsgruppen von praktischer Bedeutung.

Das Ellbogengelenk (Articulatio cubiti) mit seinen 3 beteiligten Knochen (Oberarmknochen, Elle und Speiche) stellt eine komplexe Knochenverbindung der obe-

OP-JOURNAL 2012; 28: 34-40

(C) Georg Thieme Verlag KG Stuttgart · New York DOI http://dx.doi.org/10.1055/s-0032-1315005 ren Extremität dar. Es erlaubt einen umfassenden Bewegungsumfang und ist somit für die volle Funktion des Armes sehr wichtig. Aufgrund dieser Tatsache und der diversen Verletzungsmuster an diesem Gelenk ist eine genaue Kenntnis der anatomischen Gegebenheiten im Rahmen der adäquaten Frakturversorgung essenziell.

Besonders im Kindesalter ist eine Wiederherstellung der Anatomie essenziell, um langfristigen Funktionseinschränkungen, Wachstumsstörungen und vorzeitigem Gelenksverschleiß vorzubeugen [2].

\section{Anatomie des kindlichen Ellbogens}

Am Ellbogengelenk sind die 3 langen Röhrenknochen (Humerus, Radius und Ulna) des Armes beteiligt. Diese bilden insgesamt 3 Gelenke, welche in der gemeinsamen Kapsel des zusammengesetzten Ellbogengelenks zu liegen kommen. Das untere Ende des Oberarmknochens ist an seiner Vorder- und Hinterseite abgeflacht. Am Ende trägt es einen Gelenkknorren (Condylus humeri), der am Ellbogengelenk beteiligt ist. Am distalen Oberarmknochen findet man das Humerusköpfchen (Capitulum humeri), welches mit der Gelenkgrube am Speichenkopf (Caput radii) in Verbindung steht und das Humeroradialgelenk bildet. Medial davon bildet der Oberarmknochen eine Gelenksrolle aus (Trochlea humeri), den Gelenkskopf des Schaniergelenks mit der Elle. Eine korrespondierende Einziehung an der Ulna (Incisura trochlearis) steht ihr als Gelenksfläche gegenüber. Zwischen den beiden Knochen des Unterarms findet sich das proximale Radioulnargelenk, welches für 


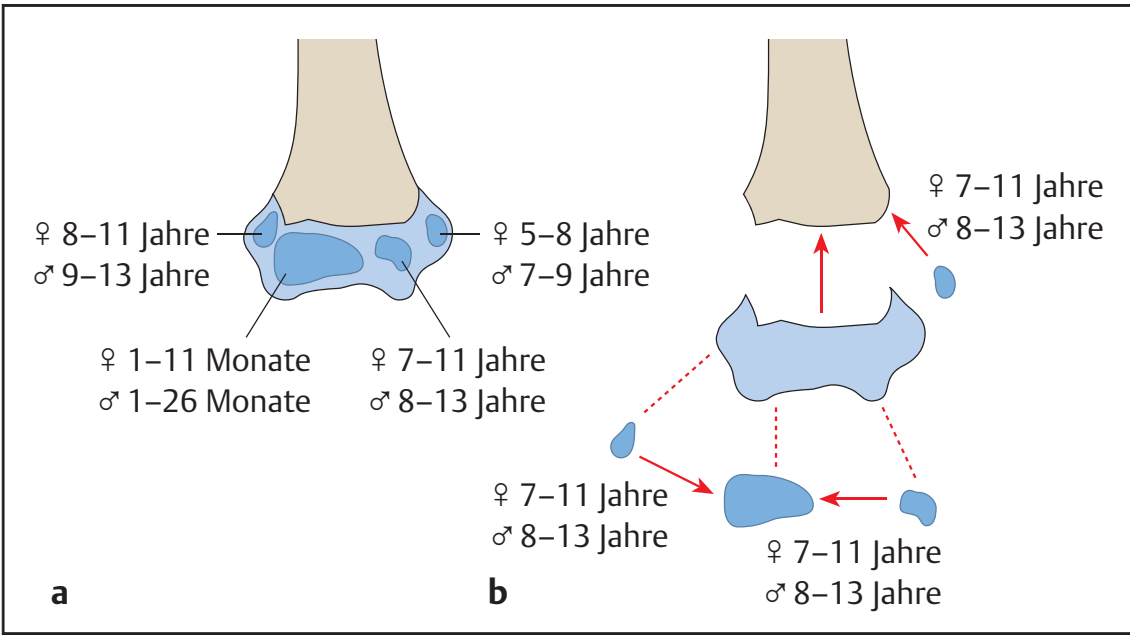

Abb. $1 \mathbf{a}$ und $\mathbf{b}$ a Zeitpunkt des Auftretens der Ossifikationskerne. b Zeitpunkt des Fugenschlusses. (Aus: Weinberg, Tscherne [Hrsg.]. Unfallchirurgie im Kindesalter - Teil 1: Allgemeiner Teil, Kopf, Obere Extremität. 2006. Mit freundlicher Genehmigung des Springer-Verlages, Heidelberg.)

die Umwendebewegungen (Pro- und Supination) von großer Bedeutung ist. Ein Ringband umschließt das Radiusköpfchen und trägt wesentlich zur Stabilität bei.

Blickt man auf das Röntgenbild eines kindlichen Ellbogens, fallen neben den erwähnten knöchernen Strukturen noch einzelne isolierte Verknöcherungskerne auf.

Von diesen geht die schrittweise Mineralisierung der knorpeligen Knochenanlage aus, welche eine entscheidende Rolle im Wachstum spielt. Deren Kenntnis ist im Rahmen der Frakturdiagnostik essenziell. So finden sich am distalen Oberarm 4 sog. Ossifikationszentren, wobei es erst im Alter von 17 Jahren zu einem vollständigen Verschluss der dazwischenliegenden Fugen und somit zum vollkommenen Abschluss des Wachstums kommt (Abb. 1). Seitlich des Gelenkknorrens am Oberarmknochen findet sich jeweils ein markanter Knochenpunkt (Epicondylus lateralis und Epicondylus medialis), welcher gut unter der Haut zu tasten ist. Diese und die Kondylen bzw. deren entsprechende Verknöcherungszonen können von Frakturen betroffen sein.

Bei Ellbogenfakturen handelt es sich um die periphersten Frakturen am Oberarmknochen. Bei der Fragestellung nach der korrekten Therapieoption für unterschiedliche Frakturtypen muss berücksichtigt werden, dass die distale Wachstumsfuge am Humerus nur eine sehr geringe Wachstumspotenz besitzt und somit nur wenig Korrekturpotenzial bez.
Achsfehlstellungen aufweist. Deshalb können bereits ab dem Alter von 7 Jahren keine Korrekturen in Frontal- und Sagittalebene mehr stattfinden. Ab diesem Alter findet im Wesentlichen nur noch das Wachstum der Form statt. Eine der physiologischen Anatomie entsprechende Stellung muss letztlich immer erzielt werden.

\section{Frakturen am Ellbogen}

Grundsätzlich gilt es, Frakturen ohne Gelenksbeteiligung von jenen mit Beteiligung der Gelenksflächen zu unterscheiden. Weitere wesentliche Kriterien in der Beurteilung von Frakturen am Ellbogen stellen das Ausmaß der Dislokation und die Beteiligung von Wachstumszonen dar. Aufgrund der niedrigen Wachstumspotenz und dem entsprechend geringen Korrekturpotenzial ist die Beurteilung der Stellung ein weiteres bedeutendes Entscheidungskriterium in oder konservativen Therapie. Abhängig vom Frakturtyp kann zwischen einer Vielzahl von Therapieoptionen gewählt werden. Konservativ versorgt werden können eher Frakturen ohne Gelenksbeteiligung und ohne wesentliche DisFrakturen, insbesondere Frakturen mit Achsfehlstellungen, sowie Frakturen, welche die Gelenksflächen betreffen, bedürfen öfters einer anatomischen Reposition und osteosynthetischen Fixierung.

Eine im Kindesalter häufige Fraktur im Bereich des Ellbogengelenks ohne Gelenksbeteiligung stellt die suprakondyläre Fraktur am distalen Oberarm dar. der Indikationsstellung zur operativen lokation. Dislozierte oder gar instabile
Kondyläre Frakturen können entweder nur einen der beiden Kondylen oder als transkondyläre Frakturen mit häufig mehreren Fragmenten die gesamte Breite des gelenksbildenden distalen Oberarms betreffen. Ellbogenluxationen gehen häufig mit komplexen Verletzungsmustern und knöchernen Abrissen einher. Am proximalen Unterarm findet man Frakturen des Radiusköpfchens an der Speiche sowie Frakturen des Olekranons an der Elle.

\section{Diagnostik}

Die Röntgenaufnahme mit anterior-posteriorem und seitlichem Strahlengang erlaubt in den allermeisten Fällen eine adäquate Diagnosestellung. Schichtbilduntersuchungen, wie CT und MR, können in Ausnahmefällen zur genaueren Frakturbeurteilung herangezogen werden. Es ist jedoch $\mathrm{zu}$ bedenken, dass diese meist bei Kindern nur unter entsprechender Sedierung möglich sind und die Computertomografie zusätzlich mit einer großen Strahlenbelastung verbunden ist. Der Strahlenschutz ist im Kindesalter aufgrund der potenziellen langfristigen Schäden von enormer Bedeutung und muss auch bei der intraoperativen Durchleuchtung bedacht werden.

Aufgrund der engen Beziehung von Gefäßen und Nerven zu den knöchernen Strukturen ist bei dislozierten Frakturen immer auch an eine Verletzung derselben zu denken und die Funktion der Strukturen zu überprüfen.

Bei eingeschränkter peripherer Durchblutung kann häufig mittels Ultraschall das Ausmaß einer Gefäßverletzung beurteilt werden. Kontrastmitteluntersuchungen der Gefäße können weiterführend zum Einsatz kommen.

\section{Operative Versorgungsmöglichkeiten}

Grundsätzlich sind 4 verschiedene osteosynthetische Verfahren, welche im Kindesalter zum Einsatz kommen können, zu unterscheiden. Neben der auch aus der Erwachsenenmedizin bekannten Verschraubung oder Verplattung gibt es noch die Möglichkeit der Frakturversorgung mittels Bohrdrähten oder elastisch stabiler Marknagelung (EMSN). Jede der genannten Therapieoptionen hat bei bestimmten Frakturen ihre Berechtigung. Die Verschraubung, die bspw. bei knöchernen Ausrissen eines Kondyls zum Einsatz kommt, und die Verplattung, welche die Versorgung von Trümmer- 


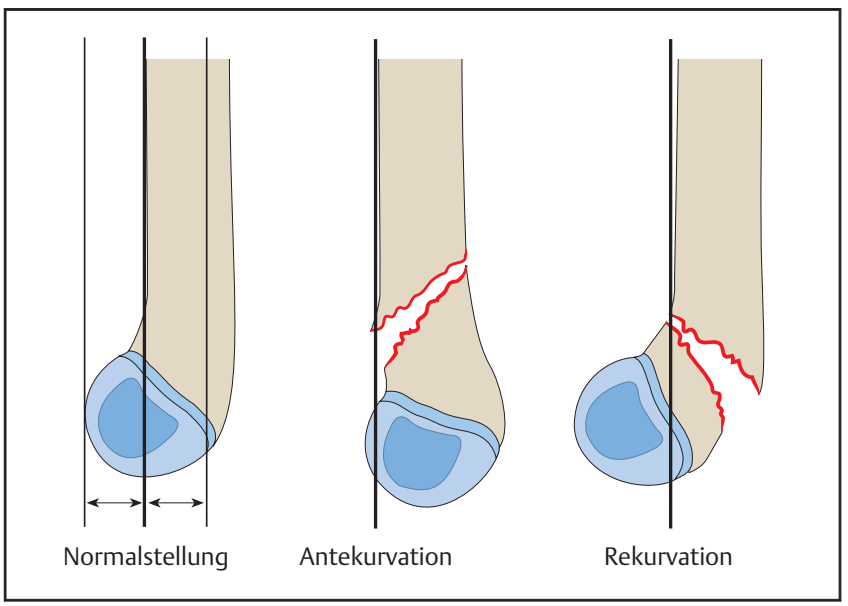

Abb.2 Radiologische Hilfslinie nach Roger: Diese beschreibt im seitlichen Strahlengang eine Tangentiale entlang der vorderen Humeruskortikalis, deren Verlängerung im Normalfall das Capitulum humeri am Übergang vom mittleren zum hinteren Drittel schneidet. (Aus: Weinberg, Tscherne [Hrsg.]. Unfallchirurgie im Kindesalter - Teil 1: Allgemeiner Teil, Kopf, Obere Extremität. 2006. Mit freundlicher Genehmigung des Springer-Verlages, Heidelberg.)

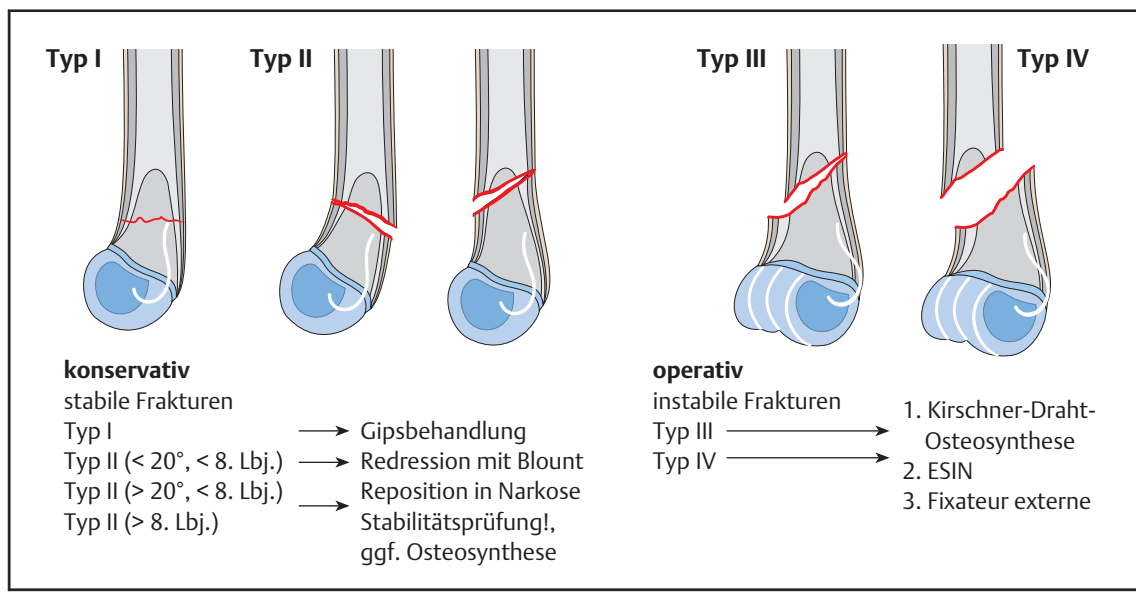

Abb. 4 Klassifikation der suprakondylären Humerusfrakturen und Therapiealgorithmus. (Aus: Weinberg, Tscherne [Hrsg.]. Unfallchirurgie im Kindesalter - Teil 1: Allgemeiner Teil, Kopf, Obere Extremität. 2006. Mit freundlicher Genehmigung des Springer-Verlages, Heidelberg.)

brüchen erlaubt, stellen stabile Osteosynthesen dar. Die elastisch stabile Marknagelung (EMSN, auch elastisch stabile intramedulläre Nagelung, ESIN, oder elastisch stabile Markraumschienung, ESMS) hingegen ist eine Bruchversorgung, die Mikrobewegungen zulässt, welche die Bruchheilung fördern können. Sie kann jedoch nur bei Frakturen im Schaftbereich zum Einsatz kommen und erlaubt keine adäquate Versorgung bei Gelenksfrakturen. Bohrdrähte können insbesondere da verwendet werden, wo Schrauben keinen Platz finden, häufig also bei kleineren Kindern. Diese bieten jedoch weit weniger Stabilität und bedürfen häufig einer postoperativen Ruhigstellung im Gips.

Als Nahtmaterial kann in der Behandlung von Kindern meist resorbierbares Material zum Einsatz kommen, wodurch den Kindern eine Nahtentfernung und damit verbundener Stress erspart werden kann.

Auf die einzelnen Indikationen für die Osteosyntheseverfahren soll im folgenden spezifischen Teil zu den unterschiedlichen Frakturen eingegangen werden. Unabhängig davon, welches Verfahren zum Einsatz kommt, ist im Kindesalter zu bedenken, dass am wachsenden Skelett eine Metallentfernung meist notwendig wird.

\section{Suprakondyläre Frakturen}

Die suprakondyläre Oberarmfraktur stellt im eigentlichen Sinn keine Gelenksfraktur dar. Aufgrund ihrer unmittelbaren Nähe zum Ellbogengelenk führt sie bei inadäquater Therapie jedoch häufig zu funktionellen Einschränkungen

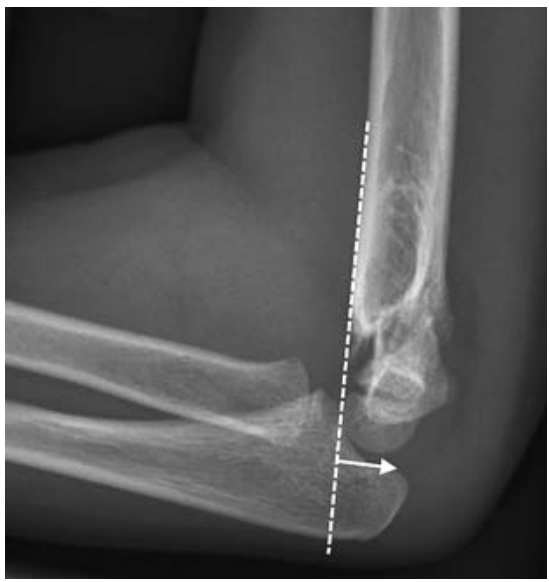

Abb. 3 Röntgenbild eines 6-jährigen Mädchens mit suprakondylärer Extensionsfraktur.

oder kosmetisch störenden Ergebnissen. Um dann den vollen Bewegungsumfang des Ellbogengelenks wiederherzustellen, können aufwendige Korrekturoperationen notwendig werden [3].

70-90\% der Ellbogenfrakturen betreffen den distalen Oberarmknochen, wobei die suprakondylären Frakturen mit einem Anteil von 60-70\% am häufigsten vorkommen.

Ausgehend vom Verletzungsmuster sind 2 Frakturtypen zu unterscheiden: Zum einen kann es bei Sturz und einem direkten Trauma auf die Rückseite des Ellbogens bei gebeugtem Arm zu einer Fraktur kommen. Hierbei verschiebt sich das distale Fragment nach vorne, es kommt also zu einer Flexionsstellung. Von diesem Flexionstyp wird der Extensionstyp unterschieden. Hierbei kommt es durch einen Sturz auf den gestreckten Arm zu einer indirekten Kraftausübung auf das Ellbogengelenk. Das Knochenfragment wird nach hinten, in eine Extensionsstellung verschoben. Eine seitliche Röntgenaufnahme des Ellbogens erlaubt eine Beurteilung der Stellung. Die vordere Kortikalis des Humerus und das Kapitulum (Gelenksköpfchen am Oberarmknochen) dienen als Referenzpunkte (Abb.2 und 3). Der Frakturtyp und die Dislokation können nach unterschiedlichen Klassifikationen eingeteilt werden, wobei v. Laer 4 Typen unterscheidet (Abb. 4). Für die Praxis relevant ist die Kenntnis jener Frakturen, die einer operativen Therapie bedürfen. Es werden stabile Frakturen, Typ I und II, welche konservativ versorgt werden können, von den instabilen Frakturen, die eine Dislokation in zumindest 2 Ebenen aufweisen, unterschieden. 


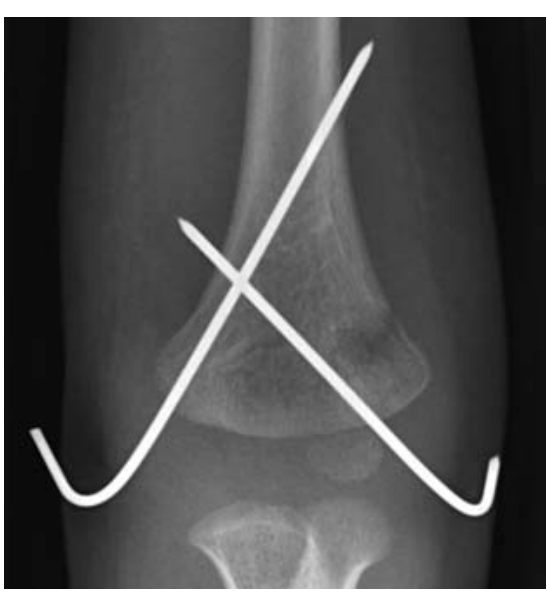

Abb. 5 Röntgenbild eines 4-jährigen Buben mit suprakondylärer Oberarmfraktur, welche mit perkutanen Bohrdrähten versorgt wurde.

Bei größeren Dislokationen ist immer auch an die Gefahr einer Gefäß- oder Nervenverletzung zu denken. Die Reposition kann offen oder geschlossen erfolgen. Bei offener Reposition wird meist ein radialer oder dorsaler Zugang gewählt. Bei Gefäß-Nerven-Verletzungen richtet sich der Zugang nach den betroffenen Strukturen, wobei meist ein ventrales Zugehen notwendig wird. Die Rückenlagerung hat sich an unserer Klinik bewährt.

Zur Frakturversorgung kommen Kirschner-Drähte, ESIN und Fixateur externe infrage. Die Kirschner-Draht-Versorgung ist eine etablierte Versorgungsmöglichkeit. Nach offener oder geschlossener Reposition werden 2 Bohrdrähte perkutan von radial und ulnar distal gekreuzt in die beiden Kondylenpfeiler eingebracht, wobei darauf zu achten ist, dass diese sich oberhalb der Frakturlinie kreuzen, um eine optimale Stabilität zu erreichen (Abb. 5). Alternativ können beide Drähte von radial-distal divergierend eingebracht werden. In jedem Fall ist auf die Schonung der N. ulnaris und radialis zu achten!

Bei der elastisch stabilen Marknagelung (ESIN) werden 2 intramedulläre Drähte von proximal in den Oberarmknochen eingebracht und das Knochenfragment anschließend nach Reposition aufgefädelt. Diese Versorgung erlaubt eine frühfunktionelle Behandlung. Der Einbringungspunkt am Oberarm liegt am proximalen Oberarmknochen distal des Tuberculum deltoideum. Die Kortikalis wird mit einem Pfriem perforiert und die vorgebogenen Drähte eingebracht. Diese werden mithilfe des T-Griffs unter Bildwandlerkontrolle in den Markraum
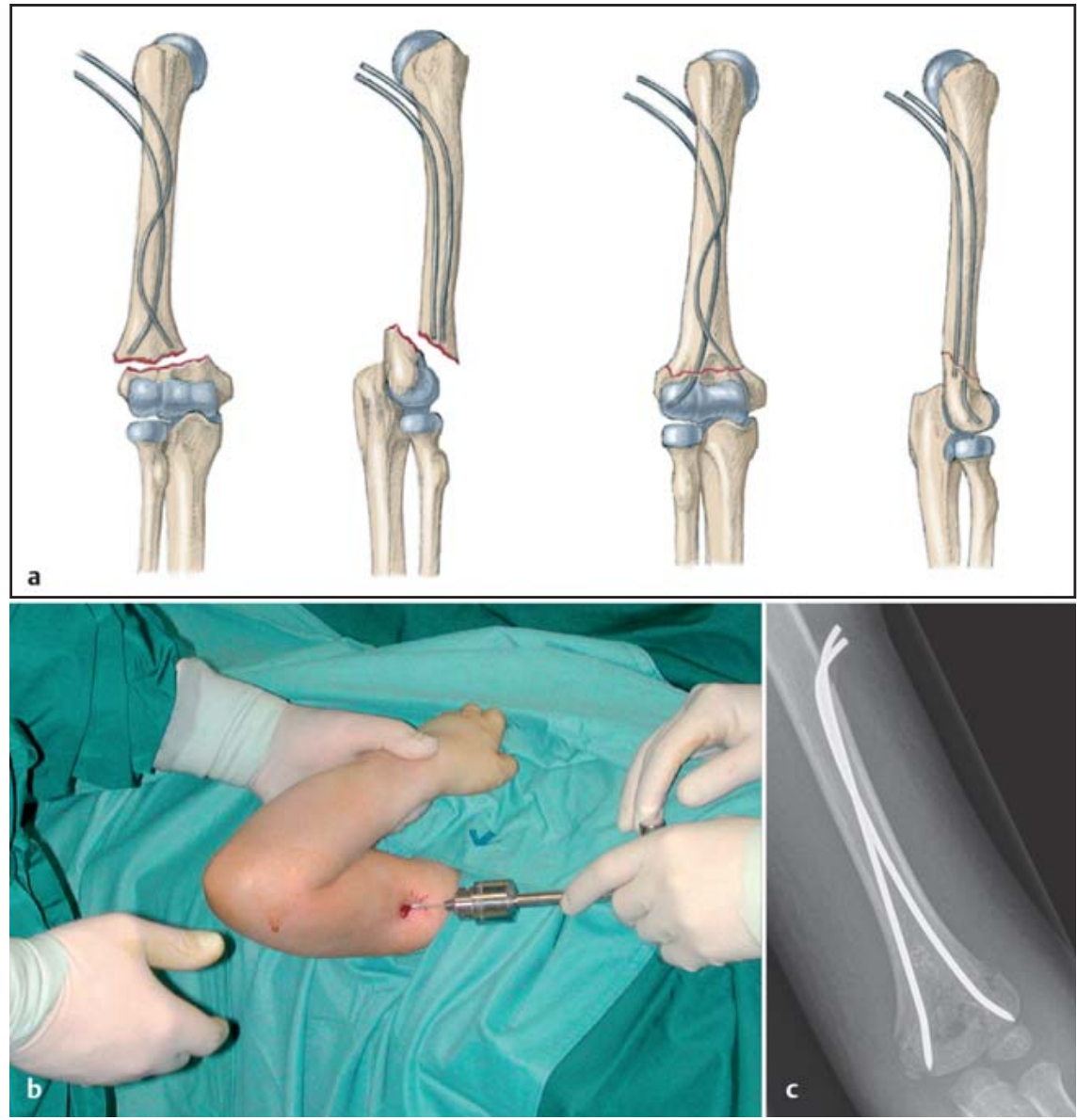

Abb. 6 a bis c a Schematische Darstellung der Versorgung von suprakondylären Oberarmfrakturen mit der intramedullären Markraumschienung. (Aus: Weinberg, Tscherne [Hrsg.]. Unfallchirurgie im Kindesalter - Teil 1: Allgemeiner Teil, Kopf, Obere Extremität. 2006. Mit freundlicher Genehmigung des Springer-Verlages, Heidelberg.) b Versorgung im OP-Saal, der Nagel wird am proximalen Oberarm eingebracht. c Röntgenbild einer suprakondylären Fraktur, welche mittels deszendierender Nagelung versorgt wird.

vorgeschoben und im distalen Knochenfragment verankert (Abb. 6).

Die Indikation zum Einsatz eines Fixateur externe kann bei Trümmerzonen gegeben sein, die sonst keine stabile Frakturversorgung erlauben. Die Anwendung sehen wir vor allem bei großem metaphysären Fragment und älteren Kindern.

Die Versorgung mit ESIN und Fixateur externe erlaubt üblicherweise eine gipsfreie Nachbehandlung, beim Einsatz von Kirschner-Drähten empfiehlt sich jedoch eine Ruhigstellung, um sekundären Dislokationen vorzubeugen. Jedenfalls sollten radiologische Kontrollen durchgeführt werden. Patienten, die eine Nervenschädigung, sei es durch den Unfall oder sekundär im Rahmen der Versorgung, erleiden, weisen meist eine gute Prognose auf. Die meisten Nervenläsionen sind nur vorübergehend, bestehen diese jedoch länger als 3 Monate, sind
Nachtlagerungsschienen induziert, um muskulären Funktionseinschränkungen vorzubeugen.

\section{Kondyläre Frakturen}

Frakturen des Condylus radialis machen $10-20 \%$ aller Frakturen am distalen $\mathrm{Hu}-$ merus aus. Es handelt sich hierbei um fugenkreuzende Gelenkfrakturen des lateralen Kondylus. Der Condylus ulnaris ist nur in seltenen Fällen isoliert betroffen, die Versorgungstrategien richten sich nach denen der Condylus-radialis-Frakturen. Diese Frakturen können in Kombination mit einer Ellbogenluxation auftreten. Das Ossifikationszentrum des lateralen Kondylus erscheint durchschnittlich um das Ende des 1. Lebensjahrs. Frakturen in diesem Bereich treten typischerweise nur am wachsenden Skelett auf, im Erwachsenenalter findet man sie kaum. 


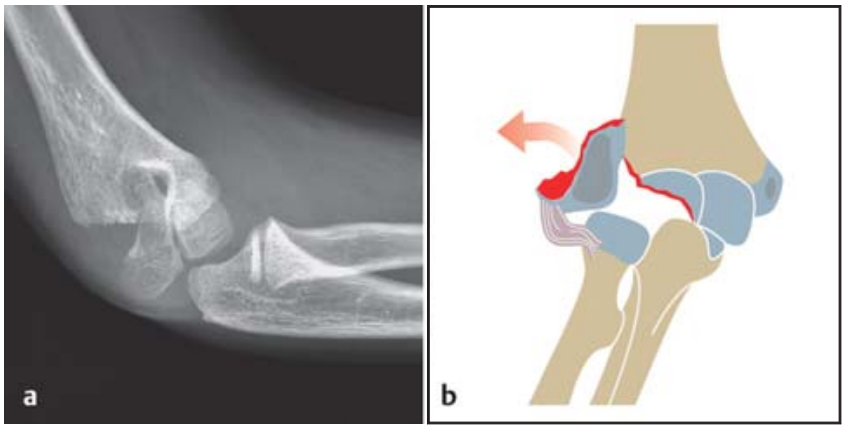

Abb. $7 \mathrm{a}$ und $\mathbf{b}$

a Röntgenbild eines 10-jährigen Buben mit Condylus-radialisFraktur. b Schema der Fraktur. (Aus: Weinberg, Tscherne [Hrsg.]. Unfallchirurgie im Kindesalter Teil 1: Allgemeiner Teil, Kopf, Obere Extremität. 2006. Mit freundlicher Genehmigung des SpringerVerlages, Heidelberg.)

Aufgrund des engen Bezugs zu der Wachstumszone können nach diesen Frakturen eine Vielzahl von Wachstumsstörungen und Komplikationen auftreten, wobei die selten auftretenden Pseudarthrosen hier genannt werden müssen. Eine adäquate Frakturversorgung ist daher von größter Bedeutung [4].

Die Diagnostik stützt sich wiederum primär auf das Nativröntgen, wobei dislozierte Frakturen leicht zu erkennen sind (Abb. 7). Bei undislozierten Frakturen kann jedoch die Unterscheidung zwischen inkompletten und kompletten Gelenksfrakturen mit der Gefahr der sekundären Dislokation mitunter schwierig sein. Es sollte daher bei einer primär konservativ versorgten Fraktur immer eine kurzfristige radiologische Kontrolle ohne Gips erfolgen, um die Stellung zu überprüfen.

Die Indikation zur operativen Therapie wird bei Sekundärdislokationen sowie primär dislozierten Frakturen gesehen. Die vollständig verschobene Fraktur des radialen Kondylus ist eine Gelenksfraktur und eine rasche osteosynthetische Versorgung ist indiziert. Als Versorgungsmaterial kommen Kleinfragmentzugschrauben mit Unterlegscheiben und kanülierte Schrauben aus Titan zum Einsatz. Hierbei sollte auf die Gewindelänge geachtet werden, sodass dieses nicht im Frakturspalt zu liegen kommt. Der Patient wird in Rückenlage gelagert, der Arm soll frei beweglich abgedeckt werden. Es wird ein lateraler Zugang zum Ellbogengelenk gewählt und die Fraktur unter Sicht reponiert und osteosynthetisch versorgt. Bei Kindern < 5 Jahren kann das Frakturfragment für eine Schraubenversorgung mitunter zu klein sein. Hier kann eine Kirschner-Draht-Fixierung erfolgen, die jedoch Nachteile bez. der Kompression und Stabilität aufweisen kann. Mittels Bohrdraht versorgte Frakturen benötigen wiederum einer Ruhigstellung in einer Gipsschale.
Aufgrund der potenziellen Wachstumsstörungen mit entsprechenden Varusund Valgusdeformitäten empfiehlt es sich, betroffene Kinder über einen längeren Zeitraum zu kontrollieren.

\section{Transkondyläre Frakturen}

Die transkondylären Frakturen sind äußerst seltene Frakturen des körperfernen Oberarmendes, wobei sich ein Häufigkeitsgipfel um den Wachstumsabschluss zeigt. Die radiologische Basisdiagnostik in 2 Ebenen erlaubt meist eine adäquate Diagnosestellung. Bei nur geringer Dislokation kann mitunter eine Unterscheidung von einer suprakondylären Fraktur erschwert sein. Bei Jugendlichen mit Mehrfragmentfrakturen kann die 3-dimensionale Rekonstruktion mittels CT zur Operationsplanung hilfreich sein.

Primäres Ziel jeder Therapie muss es sein, die Integrität der Gelenksfläche wiederherzustellen. Bei verschobenen Brüchen kann dies meist nur mittels Operation den unikondylären Frakturen kommen auch hier wiederum Schrauben und Bohrdrähte in Abhängigkeit vom Alter des Patienten zum Einsatz. Meist kann jedoch nur in Kombination mit einer Verplattung eine ausreichende Stabilität erzielt werden. Typischerweise wird ein dorsaler Zugang gewählt, wobei durch eine Olekranonosteotomie ein besserer Überblick erreicht werden kann. Dies ist meist aber nur bei älteren Kindern vonnöten. Je nachdem, ob sich intraoperativ stabile Verhältnisse zeigen, kann auf eine Ruhigstellung im Gips verzichtet werden.

\section{Ellbogenluxationen}

Ellbogenluxationen treten am häufigsten in der 2. Lebensdekade, zwischen dem 10. und 20. Lebensjahr, auf. bewerkstelligt werden. Ähnlich wie bei

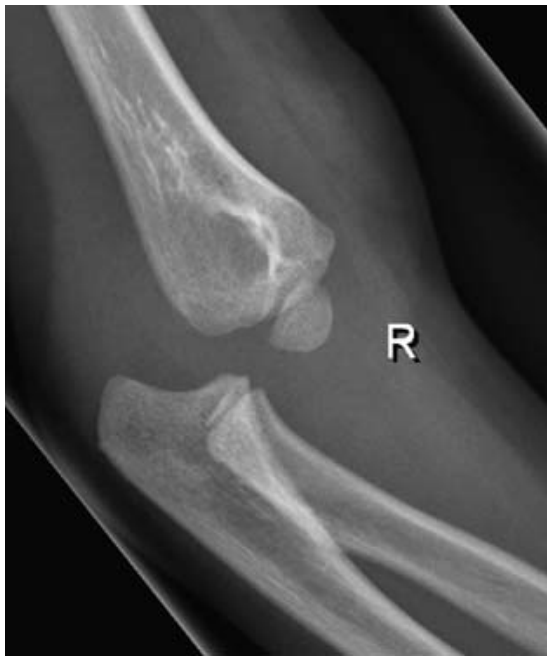

Abb. 8 Röntgenbild eines 7-jährigen Buben mit Ellbogenluxation.

Grundsätzlich muss mit knöchernen Begleitverletzungen gerechnet werden, die in bis zu 90\% der Fälle auftreten [5].

Am häufigsten findet man epikondyläre Abrissverletzungen, wobei der ulnare Epikondylus häufiger betroffen ist. Es handelt sich hierbei um eine Avulsionsfraktur, wobei es durch den Valgusstress zu einem Zug über die dort entspringenden Bandstrukturen und damit einem knöchernen Ausriss kommt. Auch im Bereich der Epikondylen finden sich Verknöcherungskerne, die eine Darstellung einer undislozierten Fraktur erschweren können. Anders als bei den Brüchen der Kondylen sind die Apophysenverletzungen komplikationsärmer, da diese nicht zum Längenwachstum beitragen.

Abhängig von der Richtung der Verrenkung können unterschiedliche Typen der Ellbogenluxation unterschieden werden. Die Diagnose kann in den meisten Fällen mit einem Röntgenbild in nur 1 Ebene gestellt werden (Abb. 8).

Die Ellbogenluxation muss jedenfalls notfallmäßig reponiert werden. Eine anschließende radiologische Bildgebung in den Standardebenen kann helfen, weitere Verletzungen darzustellen. Nach Reposition erfolgt die Prüfung der Stabilität. Im Kindesalter wird ggf. großzügig eine Ruhigstellung durchgeführt, um die Bandheilung zu gewähren. Im Erwachsenenalter müssen diese v.a. radial refixiert werden.

Die Indikation zur operativen Versorgung einer Epicondylus-ulnaris-Abrissfraktur stellt sich bei dislozierten Brüchen, ins- 


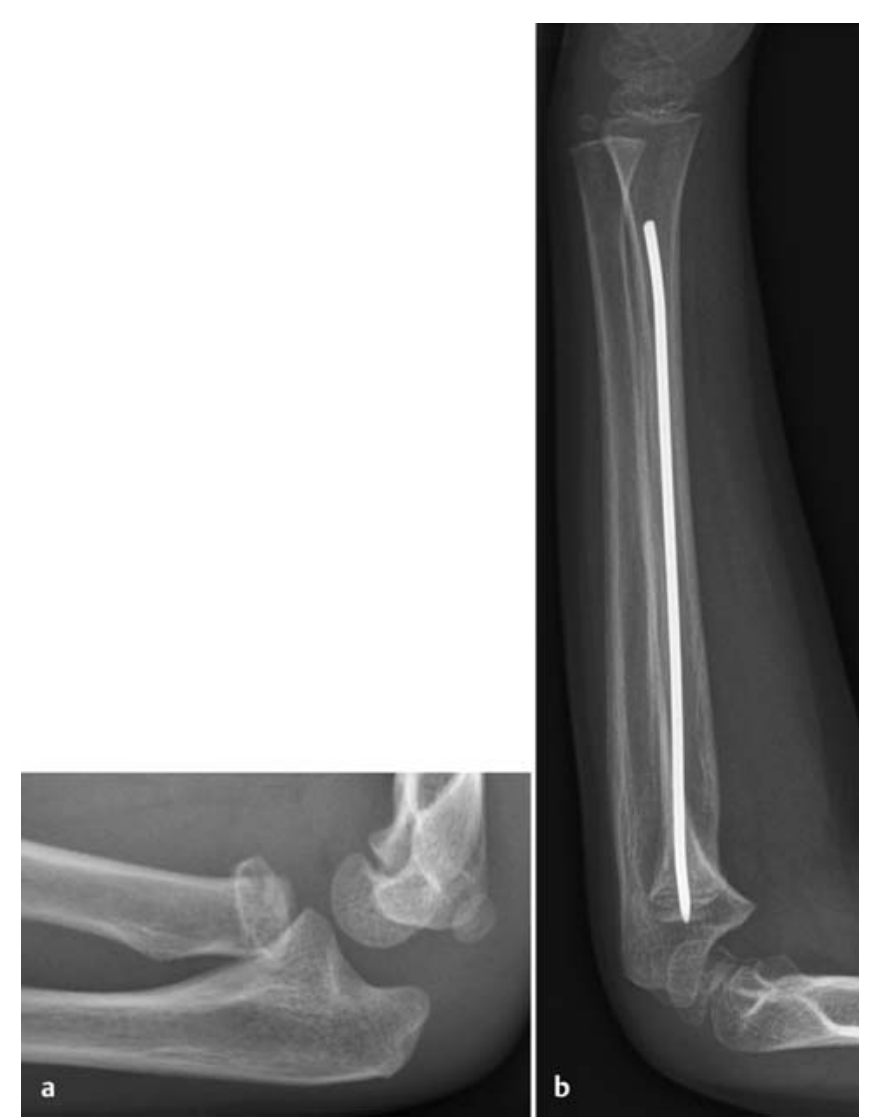

besondere bei N.-ulnaris-Läsionen, sowie bei nach intraartikulär eingeschlagenem Fragment. Ziel des operativen Vorgehens ist eine anatomische und sichere Refixation des abgerissenen Epikondylus und damit der daran entspringenden Muskeln und Seitenbandstrukturen. Als ideal ist eine Schraubenosteosynthese zu bewerten, da diese eine funktionelle Therapie erlaubt. Die offene Reposition erfolgt über einen kleinen ulnaren $\mathrm{Zu}$ gang. Der Patient befindet sich in Rückenlage, der Arm soll frei beweglich sein. Besondere Vorsicht gilt bei dieser Operation dem N. ulnaris, der vor iatrogener Schädigung geschützt werden muss.

\section{Radiusköpfchenfrakturen}

Radiusköpfchen- und Radiushalsfrakturen finden sich im Kindes- und Jugendalter nur selten, sie machen etwa $1 \%$ aller Extremitätenfrakturen aus. Da das Radiusköpfchen bei Kindern noch fast vollständig aus Knorpel besteht, sind Trümmerfrakturen in diesem Bereich nicht zu erwarten. Am häufigsten finden sich subkapitale Frakturen und Epiphysenlösungen. Im Röntgenbild lässt sich der Verknöcherungskern des Radiusköpfchens erst in einem Alter von etwa
Abb. 9 a und $b$

a Röntgenbild eines 5-jährigen Mädchens mit Radiushalsfraktur, b mittels ESMN reponiert.

5-7 Jahren nachweisen. Verletzungen in diesem Bereich können zu vorzeitigem Fugenschluss und Wachstumsverzögerung führen. Therapieziel ist die Wiederherstellung der vollen Funktion, eine schonende Behandlungsstrategie ist hierfür unumgänglich.

Abhängig vom Alter kann ein unterschiedliches Ausmaß einer Verkippung des Radiusköpfchens toleriert werden. Bei jüngeren Patienten ist aufgrund des noch größeren Wachstumspotenzials eher eine Spontankorrektur zu erwarten. Ist damit nicht mehr zu rechnen, muss eine Reposition erfolgen, wobei der geschlossenen Reposition der Vorzug zu geben ist. Die offene Reposition kann mit Weichteilverletzungen und Vernarbungen einhergehen, welche wiederum ein Funktionsdefizit nach sich ziehen. Gelingt eine geschlossene Reposition nicht, kann ein intramedullärer Nagel in der ESIN-Technik vom distalen Radius aus eingebracht und über diesen das Fragment aufgerichtet werden (Abb.9). Dieser Nagel kann zur Schienung belassen werden, was eine frühfunktionelle gipsfreie Behandlung erlaubt.
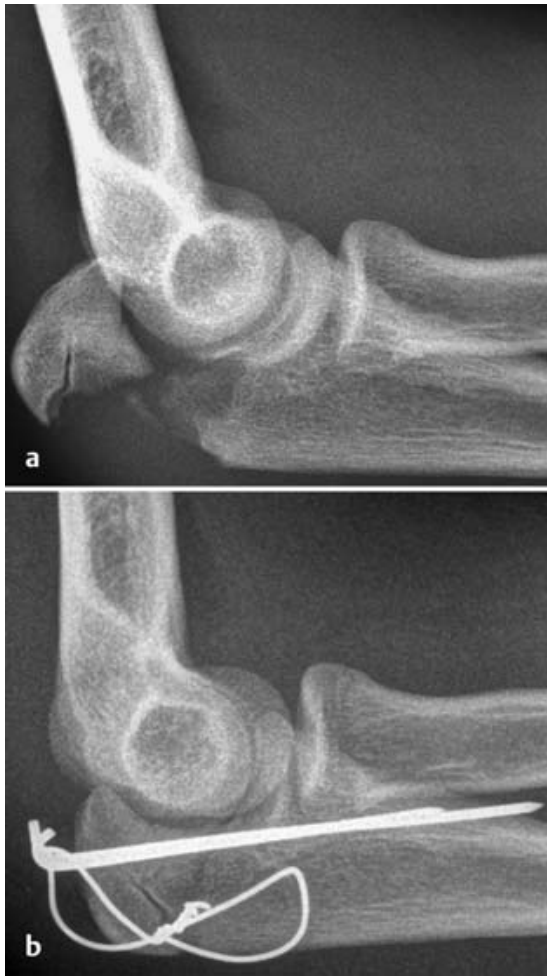

Abb. 10a und b a Röntgenbild eines 14jährigen Buben mit Olekranonfraktur, b mittels Zuggurtung versorgt.

\section{Olekranonfrakturen}

Sehr selten finden sich isolierte Olekranonbrüche. Als Unfallmechanismus kommen sowohl direkte Traumata als auch indirekte Krafteinwirkungen infragen. Wiederum gilt es zwischen intraund extraartikulären Brüchen zu unterscheiden. Das Nativröntgen ist hierbei wegweisend. Bei Brüchen mit Gelenksbeteiligung ist auf eine anatomische Reposition zu achten.

Der operative Zugang erfolgt über eine dorsale Schnittführung. Als Osteosyntheseverfahren kommen Zuggurtungen, Verplattungen und Verschraubung infrage. Die Zuggurtung stellt ein adäquates Verfahren bei Querbrüchen dar, die Verschraubung mit Zugschrauben kommt bei Schrägfrakturen zum Einsatz (Abb. 10). Bei Stückfrakturen, insbesondere bei älteren Patienten, kann eine Verplattung eine gute Stabilisierung gewährleisten. Im Gegensatz zum Erwachsenen kann bei sehr kleinen Kindern auch die alleinige parallele KD-Fixierung mit Ruhigstellung ausreichend sein.

Ebenso im Bereich des kopfnahen Endes der Elle kann es an deren Vorderseite zu Brüchen des Processus coronoideus 
kommen. Es handelt sich hierbei um eine seltene Abscherfraktur und wird häufig in Rahmen von Ellbogenluxationen beobachtet. Sie ist im Kindesalter eine Rarität. Aufgrund der nur geringen Größe des Fragments ist häufig nur eine konservative Therapie möglich. Im Rahmen einer operativen Versorgung wird der Zugang in Abhängigkeit von den Begleitverletzungen gewählt, wobei eine Verschraubung gute Stabilität gewährleistet.

\section{Postoperative Behandlung}

Ob eine Ruhigstellung nach Operation im Gips notwendig ist, muss der Chirurg abhängig vom intraoperativen Befund und der mit der Osteosynthese erzielten Stabilität entscheiden. Insgesamt kann im Kindesalter diese eher großzügig indiziert werden, da posttraumatische Bewegungseinschränkungen sich wieder aufheben. Im Ellenbogen kann dies aber durchaus auch bis zu 6 Monate dauern. Physiotherapie sollte immer im schmerzfreien Rahmen stattfinden. Man muss bedenken, dass die Einschränkung nicht auf knöcherne Fehlstellungen zurückzuführen ist. Dann muss gewartet werden, bis die Fehlstellung vom Gelenk wegwächst. Als Beispiel sei hier die Antekurvationsfehlstellung nach suprakondylären Oberarmfrakturen genannt. Gegebenenfalls wird der Gips noch in Narkose angelegt, um sekundären Dislokationen vorzubeugen. In jedem Fall ist eine postoperative Röntgenkontrolle notwendig, um die korrekte Stellung zu dokumentieren.
Die Metallentfernung muss am wachsenden Skelett im Rahmen einer 2. Narkose/Sedation erfolgen. Sie ist nach radiologisch verifizierter Knochenheilung indiziert.

\section{Zusammenfassung}

Abschließend kann festgestellt werden, dass das Ellbogengelenk in seiner physiologischen Funktion eine komplexe Struktur darstellt. Eine Vielzahl unterschiedlicher Verletzungen an den beteiligten Knochen kann nach Stürzen mit direkter oder indirekter Krafteinwirkung auftreten. Ebenso vielseitig stellen sich auch die Behandlungsoptionen dar.

Ziel muss letztlich immer sein, die volle Funktion der oberen Extremität und die Bewegung in allen Ebenen zu erhalten.

Weiters ist potenziellen Wachstumsstörungen vorzubeugen, insbesondere da die Kompetenz, Fehlstellungen zu korrigieren, bereits früh um das 7 . Lebensjahr zum Stillstand kommt und die distale Wachstumsfuge am Humerus somit zu den niedrig potenten Fugen zu rechnen ist [6].

\section{Literatur}

${ }^{1}$ Chambers HG, Wilkins KE. Fractures and Dislocations of the Elbow Region. In: Rookwood CA, Wilkins KE, Beaty JH eds. Fractures in Children. Philadelphia: Lippincott; 1996: 653-669

2 Eberl R, Weinberg AM. Posttraumatische Korrekturoperationen am kindlichen Ellenbogen. Unfallchirurg 2010; 113: 139-148

3 Weinberg AM, Marzi I, Günter SM et al. Die suprakondyläre Oberarmfraktur im Kindesalter
- eine Effizienzstudie. Ergebnisse der multizentrischen Studie der Sektion Kindertraumatologie der Deutschen Gesellschaft für Unfallchirurgie - Teil I: Epidemiologie, Effektivitätsprüfung und Klassifikation. Unfallchirurg 2002; 105: 208-216

${ }^{4}$ Haxhija EQ Mayr JM, Grechenig W et al. Treatment of medial epicondylar apophyseal avulsion injury in children. Oper Orthop Traumatol 2006; 18: 120-134

${ }^{5}$ Regel G, Weinberg AM, Seekamp A et al. Das Komplextrauma des Ellenbogen. Orthopade 1997; 26: 1020-1029

${ }^{6}$ Weinberg A, Tscherne H. Ellbogen. In: Weinberg $A$, Tscherne $H$. Unfallchirurgie im Kindesalter. Heidelberg: Springer; 2006

Dr. med. univ. Peter Ferlic Assistenzarzt

Medizinische Universität Graz

Auenbruggerplatz 34

8036 Graz

Österreich

\section{Dr. med. univ. Silvia Zötsch}

Assistenzarzt

Univ. Klinik für Kinder- und

Jugendchirurgie

Medizinische Universität Graz

Auenbruggerplatz 34

8036 Graz

Österreich

Assoz. Prof. Priv.-Doz. Dr. med. Annelie Weinberg

Chefärztin

Unfallchirurgie und Orthopädie

Mathias-Spital Rheine

Frankenbergstraße 31

48431 Rheine

annelie.weinberg@t-online.de 